\title{
Validation of a Non-Line-of-Sight Path-Loss Model for V2V Communications at Street Intersections
}

\author{
Taimoor Abbas*, Andreas Thiel ${ }^{\dagger}$, Thomas Zemen ${ }^{\ddagger}$, Christoph F. Mecklenbräuker ${ }^{\S}$, and Fredrik Tufvesson* \\ *Department of Electrical and Information Technology, Lund University, Lund, Sweden \\ ${ }^{\ddagger}$ Forschungszentrum Telekommunikation Wien (FTW), Vienna, Austria \\ ${ }^{\dagger}$ Delphi Deutschland GmbH, Bad Salzdetfurth, Germany \\ $\S$ Institute of Telecommunications, Vienna University of Technology, Vienna, Austria \\ email: taimoor.abbas@eit.lth.se
}

\begin{abstract}
In this paper a non-line-of-sight (NLOS) path-loss and fading model developed for vehicle-to-vehicle (V2V) communication at $5.9 \mathrm{GHz}$ is validated with independent and realistic measurement data. The reference NLOS model is claimed to be flexible and of low complexity, and incorporates specific geometric aspects in a closed-form expression. We validated the accuracy of the model with the help of realistic channel measurements performed in selected street intersections in the city of Lund and Malmö, Sweden. The model fits well, with a few exceptions, to the measurements. Those are in turn made in different intersections having variable geometries and scattering environments. It is found that the model can be made more general if an intersection dependent parameter, that depends on the property and number of available scatterers in that particular intersection, is included in the model.
\end{abstract}

\section{INTRODUCTION}

Intelligent transportation systems (ITS) have gained considerable attention in recent years, because they have the potential to enhance location awareness and safety of vehicles. Safetyrelated applications, e.g., cross-traffic assistance and traffic condition warnings, will help to reduce the rate of accidents. The idea is to utilize vehicle-to-vehicle (V2V) and vehicle-toinfrastructure (V2I) communications for such applications.

A lot of research efforts and field trails focusing on V2V safety applications have been done and are still ongoing both in academia and the vehicular industry. There are a number of challenges and use cases that are not yet completely understood and require further investigation. Among those, cross-traffic assistance in urban street intersections is one of the most critical use cases as visual line-of-sight (LOS) is often blocked by buildings at the corners of the streets. When the LOS is not available then scattering, i.e., reflection, diffraction and refraction, of radio waves can enable signal reception.

There exist a number of $\mathrm{V} 2 \mathrm{~V}$ measurement and ray-tracing simulation based studies to characterize the radio channel in urban street intersections [1]-[6]. In these studies it was found that the geometry of the street intersections, i.e., street width and alignment, structure of buildings, and antenna height have great impact on the NLOS reception in an urban intersection at $5.9 \mathrm{GHz}$ frequency. Moreover, it is found that $\mathrm{V} 2 \mathrm{~V}$ ray-tracing simulation models often underestimate channel parameters like, e.g., delay spread and Doppler spread, for certain streets due to limited geographical information. Thus, a generalized and well validated NLOS model is needed for urban intersections.

Based on an extensive channel measurement campaign Mangel et al. in [7] have developed a flexible and generalized NLOS path-loss model named VirtualSourcel $1 p$ at $5.9 \mathrm{GHz}$. The channel measurements were performed in many different street intersections in the city of Munich, Germany, which were selected carefully such that they may represent a majority of urban street intersections. However, it is anticipated that the given VirtualSource1 1p NLOS path-loss model can be limited in its validity to the intersections where the measurements were conducted. Thus, it would be interesting to validate the model using an independent measurement data set and study the generalizability of the model.

In this paper, we validate the afore-mentioned NLOS pathloss model using independent and realistic channel measurement data recorded in a measurement campaign called DRIVEWAY [8]. To the author's best knowledge there has been no such validation studies of the NLOS path-loss model and large-scale fading of [7] using independent measurement data.

The DRIVEWAY measurements were conducted in various traffic situations that are of particular interest for safety-related ITS applications. All test sites are located in and around the cities of Lund and Malmö in southern Sweden. The aim of our measurements was to understand and model the properties of $\mathrm{V} 2 \mathrm{~V}$ radio channels with a realistic measurement setup in realistic traffic conditions. However, in this paper, the measurement data recorded only at urban street intersections is used for the analysis.

The rest of the paper is organized as follows; section II summarizes the DRIVEWAY $\mathrm{V} 2 \mathrm{~V}$ measurement campaign where the measurement setup, scenarios description and measurement data evaluation are discussed. Section III explains very briefly the VirtualSource 11p NLOS path-loss model followed by Section IV in which the model is validated using measurement data. Finally, section V summarizes the whole discussion and concludes the paper.

\section{V2V MEASUREMENT CAMPAIGN}

The measurements were performed using standard hatchback style cars with roof mounted four-element antenna arrays, 


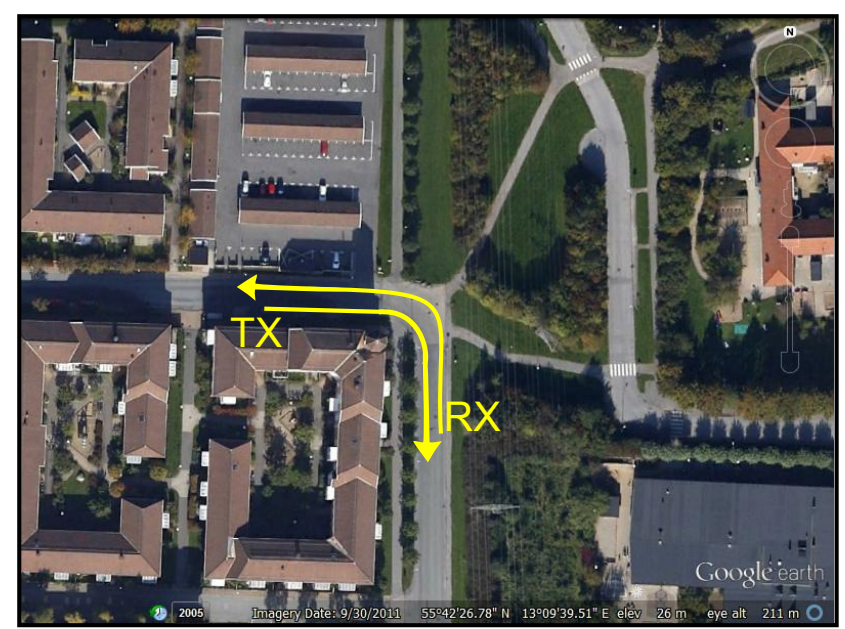

(a) Intersection-1 (N $55^{\circ} 42^{\prime} 26^{\prime \prime}$, E $13^{\circ} 9^{\prime} 39^{\prime \prime}$ )

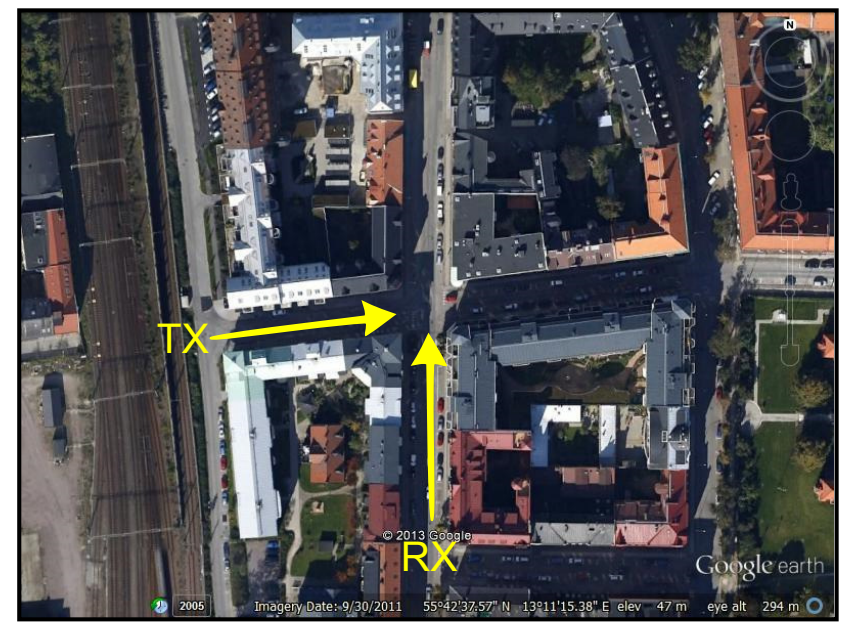

(c) Intersection-3 (N $55^{\circ} 42^{\prime} 37^{\prime \prime}$, E $13^{\circ} 11^{\prime} 15^{\prime \prime}$ )

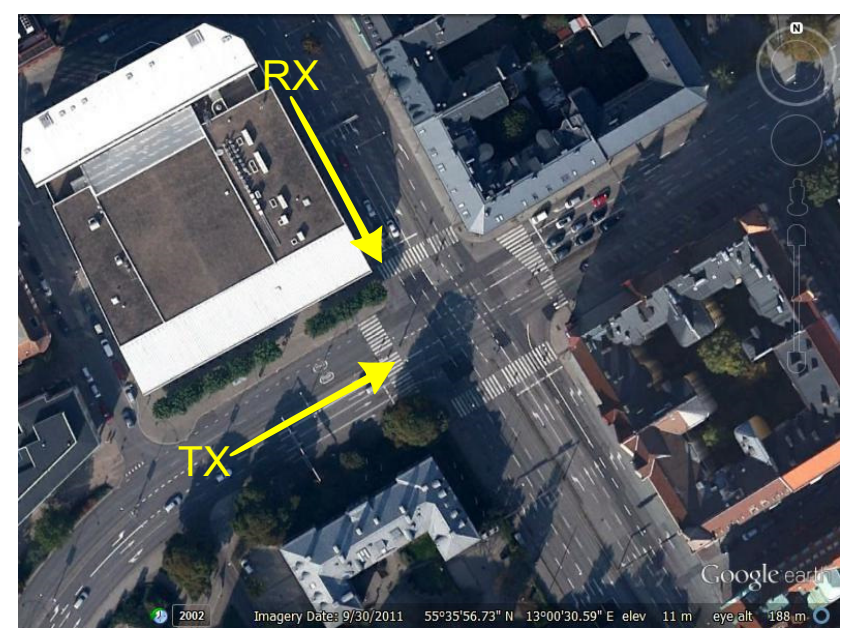

(b) Intersection-2 (N $55^{\circ} 35^{\prime} 56^{\prime \prime}$, E $13^{\circ} 0^{\prime} 30^{\prime \prime}$ )

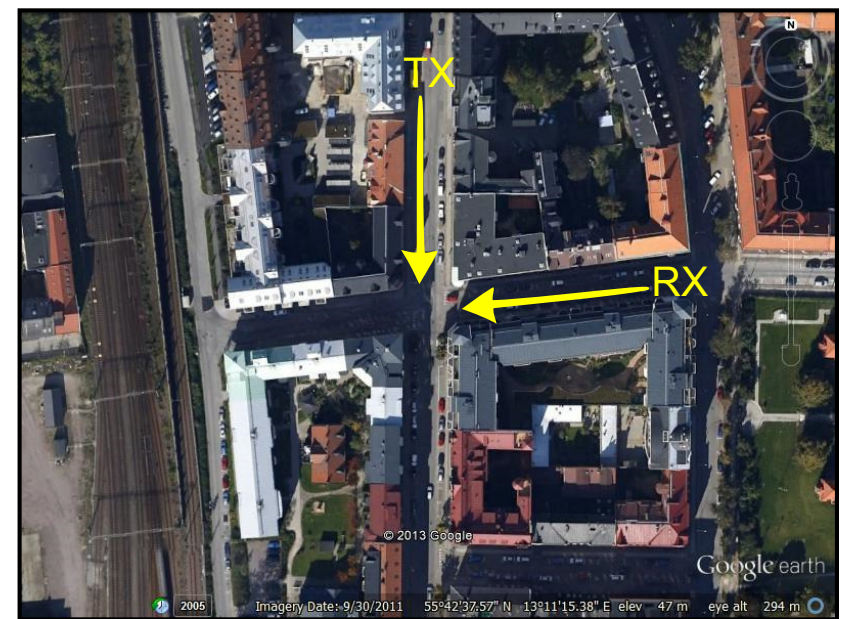

(d) Intersection-4 (N 55 $42^{\prime} 37^{\prime \prime}$, E $13^{\circ} 11^{\prime} 15^{\prime \prime}$ )

Fig. 1. Google Earth ${ }^{\mathrm{TM}}$ [9] aerial image of the four measured street intersections in Lund and Malmö. In each intersection the TX and RX vehicles were approaching the intersection center while driving along the yellow lines. All the images have same map scale.

specifically designed for V2V communication [10]. During the measurements the channel transfer function $H(f, t)$ was recorded using the RUSK Lund channel sounder that performs switched-array MIMO measurements [11]. Each transmitter (TX) and receiver (RX) vehicle logged their GPS coordinates, and videos were taken through the windshield of the TX/RX cars. In the post-processing these data were also used in combination with the measurement data. The most important measurement parameters are listed in Table I.

\section{A. Scenarios}

During the DRIVEWAY measurement campaign several measurements were recorded in many different propagation environments. The selected measurement scenarios are of particular interest for many safety related intelligent transport systems (ITS) applications [8]. In this paper we are mainly interested in NLOS propagation, especially in the intersection scenario. The reference NLOS path-loss model takes into account the inter-building distance, i.e., the street widths $w_{t}$ and $w_{r}$, on the transmitter as well as on the receiver side,
TABLE I

MEASUREMENT PARAMETERS CONFIGURATION

\begin{tabular}{ll}
\hline Parameter & Value \\
\hline Center frequency [GHz], $\mathrm{f}_{\mathrm{C}}$ & 5.6 \\
Measurement bandwidth $[\mathrm{MHz}], \mathrm{BW}$ & 240 \\
Test signal length $[\mu \mathrm{s}], \tau_{\mathrm{max}}$ & 3.2 \\
Snapshot repetition time $[\mu \mathrm{s}], \mathrm{t}_{\mathrm{rep}}$ & 307.2 \\
Number of snapshots in time, $\mathrm{N}_{\mathrm{t}}$ & 32500 or 65000 \\
Number of samples in frequency, $\mathrm{N}_{\mathrm{f}}$ & 769 \\
Recording time [s], trec & 10 or 20 \\
Number of TX antenna elements & 4 \\
Number of RX antenna elements & 4 \\
TX/RX antenna height $[\mathrm{m}]$ & 1.73 \\
Average TX plus RX antenna gain $[\mathrm{dB}]$ & 6.7 \\
Approximate system loss $[\mathrm{dB}]$ & 4 \\
\hline
\end{tabular}

distance of the TX and RX from the intersection center and TX position on the street relative to side walls. To be able to analyze the effect of these variations, we have selected 9 measurements ( 2 suburban and 7 urban) recorded in the four different street intersections of variable street widths and 
propagation condition, shown in Fig. 1. The speed of the TX and RX cars were varying between $7-11 \mathrm{~m} / \mathrm{s}(25-40 \mathrm{~km} / \mathrm{h})$, depending on the street intersection and yield signs.

Intersection-1: (N $55^{\circ} 42^{\prime} 26^{\prime \prime}$, E $13^{\circ} 9^{\prime} 39^{\prime \prime}$ ) can be described as suburban in which the buildings are situated to the left, to the right is an open surrounding with some vegetation and a power line. In both measurements the TX and RX vehicles are approaching the intersection, in $18 \mathrm{~m}$ and $20 \mathrm{~m}$ wide orthogonal streets, from south-to-north and from westto-east, respectively (see Fig. 1(a)).

Intersection-2: (N $55^{\circ} 35^{\prime} 56^{\prime \prime}$, E $13^{\circ} 0^{\prime} 30^{\prime \prime}$ ) can be described as urban with wider geometry where multi-story buildings are situated at each corner of the intersection. In the measurement the TX and RX vehicles are approaching the intersection, in $43 \mathrm{~m}$ and $23 \mathrm{~m}$ wide orthogonal streets, from southwest-to-northeast and from northwest-to-southeast, respectively (see Fig. 1(b))

Intersection-3: (N $55^{\circ} 42^{\prime} 37^{\prime \prime}$, E $\left.13^{\circ} 11^{\prime} 15^{\prime \prime}\right)$ can be described as urban with relatively narrow geometry where $3-4$ story buildings are situated at each corner of the intersection. In both measurements the TX and RX vehicles are approaching the intersection, in $20 \mathrm{~m}$ and $24 \mathrm{~m}$ wide streets, from westto-east and from south-to-north, respectively. Streets are not perfectly orthogonal. (see Fig. 1(c)).

Intersection-4: (N $55^{\circ} 42^{\prime} 37^{\prime \prime}$, E $13^{\circ} 11^{\prime} 15^{\prime \prime}$ ) is the same as intersection-3. The only difference is that the TX and RX vehicles are approaching the intersection, in $24 \mathrm{~m}$ and $23 \mathrm{~m}$ wide, from north-to-south and east-to-west, respectively. (see Fig. 1(d)).

\section{B. Data Evaluation and Results}

To analyze the measurement data we first derive the instantaneous time-varying power-delay-profile (PDP) of each measurement using the channel transfer functions at each time instant. These PDPs are averaged over the time samples that correspond to a movement of 10 wavelengths in order to remove the effect of small scale fading (for details see [1]). The temporal variations, impact of scatters as a function of propagation distance, the LOS component and other specular/non-specular components can be visualized in the averaged-PDP (APDP) plots. APDPs of two selected measurements taken at the intersection-1 and intersection-2 are shown in Figs. 2(a) and 2(b). From the APDP plot it is evident that the scattering environment is very different in both intersection-1 and 2. Intersection-2 has many MPCs including a couple of strong MPCs, due to wider streets and presence of buildings at the corners. Whereas in intersection-1 an open surrounding to one side result in fewer MPCs with less received power in the NLOS. This difference in the received power due to the geometry of the intersections can be best appreciated by looking at Fig. 2(c), in which the received power is plotted as a function of combined distance from TX to $\mathrm{RX}$ via the intersection center $\left(d_{d c}\right)$. The negative and positive $d_{d c}$ indicate that the TX and RX vehicles are approaching and leaving the intersection center, respectively. The effect of the antenna gain and cable losses have been removed from the measured channel gains. Moreover, the transmit power $\left(P_{T X}\right)$ of $23 \mathrm{dBm}$ is used that is added in the measured channel gain in order to calculate the received power $\left(P_{R X}\right)$.

To observe the overall difference in the received power due to the variable geometry and the scattering environment the received powers of the measurements at each intersection are presented together in Fig. 2(d). As a first observation, it can be seen that the smallest power is received at the Intersection1 which is logical as it has an open surrounding and fewer scatterers available that can aid NLOS reception.

Moreover, Intersection-2 (wider), 3 and 4 (narrow) have $3-4$ story buildings at each corner of the intersection with more or less similar traffic conditions, which means similar scattering environments. Ideally, the received power in a wide intersection should be higher than that in a narrow intersection given that the scattering environment is similar. However, this is not always the case, the received power in a narrow intersection can be higher than that in a wide intersection if the scattering environment in the narrow intersection is rich. This can be seen in Fig. 2(d) where Intersection-2, which is wider, has lower received power than Intersection-4. After a detailed investigation of the measurement results and having a close look at the geography of the intersections it is found that there are a couple of very strong reflected MPCs in Intersection- 4 unlike in Intersection- 2 . These strong reflections arise from the metallic window structures of a building at one corner of the intersection and give rise to the received power. Such an unexpected behavior in the power depends not only on the number of scatterers but on their material properties and geographic location. If we wish a model to be universal or most generalized then it should be able to reflect such unexpected behavior. In the subsequent analysis we would like to find out how well the NLOS path-loss model fits our measurement data and possible underlying limitations.

It is specified in the IEEE 802.11p standard [12] that a $10 \mathrm{MHz}$ frequency bandwidth will be used at $5.9 \mathrm{GHz}$ for $\mathrm{V} 2 \mathrm{~V}$ communication systems but the DRIVEWAY channel measurements were performed over a $240 \mathrm{MHz}$ bandwidth. For the measurements this larger bandwidth was selected to get a fine resolution in delay and to get better statistics of the channel, which is important for a detailed understanding. In the analysis of the measurement data, no difference in the received power is found when considering the total $240 \mathrm{MHz}$ of measured bandwidth or selected portions of $10 \mathrm{MHz}$ measured bandwidth.

\section{REFERENCE NLOS PATH-LOSS MODEL}

Before the comparison it is important to explain briefly the reference NLOS path-loss model. Mangel et. al. in [7] has performed an extensive measurement campaign and presented a realistic and a well validated NLOS path-loss model at $5.9 \mathrm{GHz}$ frequency named VirtualSource11p. The model is considered to be of low complexity, thus, enabling largescale packet level simulations in intersection scenarios. The VirtualSource11p model is obtained from a NLOS path-loss model proposed in [13] for cellular communications, which is slightly modified to correspond well to $\mathrm{V} 2 \mathrm{~V}$ measurements. The derivation of the VirtualSource11p model can be found 


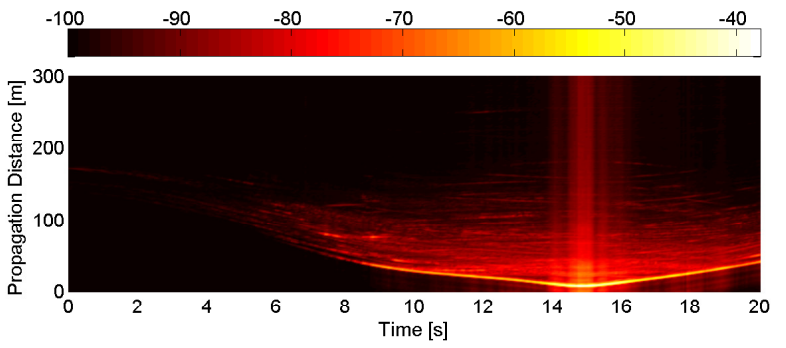

(a) APDP of Intersection-1

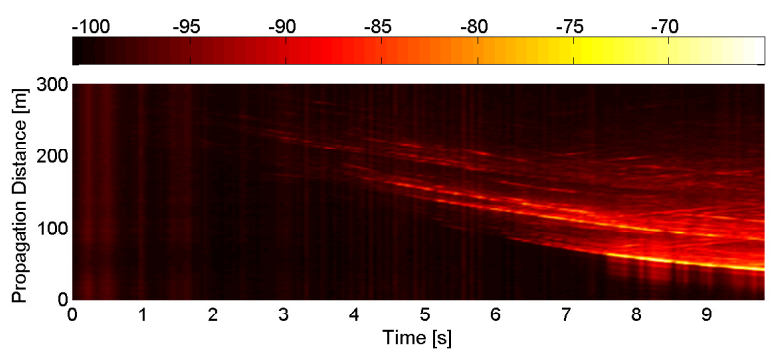

(b) APDP of Intersection-2

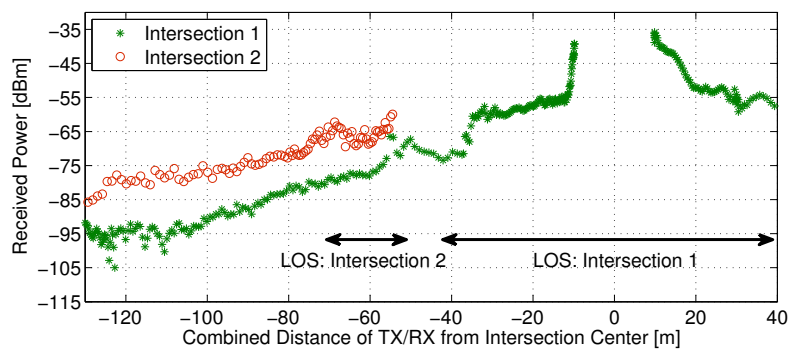

(c) Received power of Intersection-1 and 2

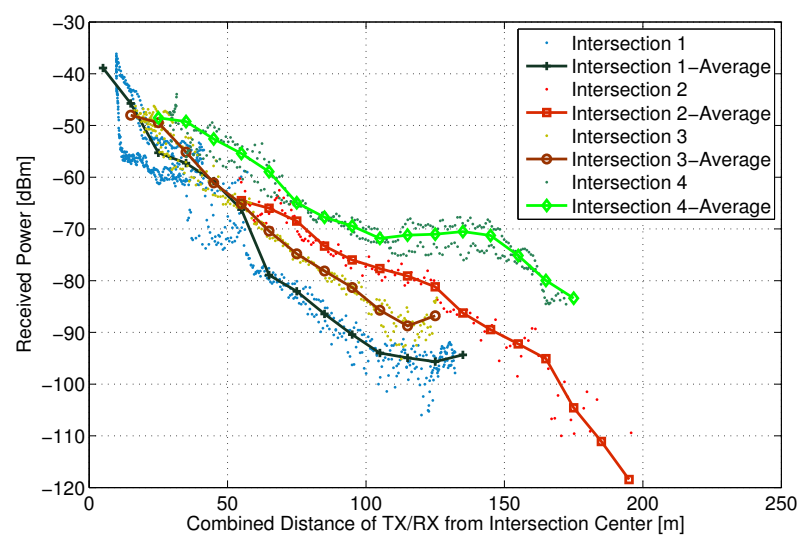

(d) Received power in all intersections

Fig. 2. Evaluation steps: a,b) APDP of 2 selected measurements at Intersection-1 and 2. The color bar indicates the received power in $\mathrm{dBm}$, c) Received power as a function of combined distance of TX/RX from the intersection center $\left(d_{d c}\right)$ for both measurements, d) Received power as function of $d_{d c}$ and curves showing their mean power averaged over every 10 meters for each intersection intersection. in [7] where the corresponding NLOS path-loss equation to calculate the received power at a certain distance is given as follows,

$$
\begin{gathered}
P L\left(d_{r}, d_{t}, w_{r}, x_{t}, i_{s}\right)=C+i_{s} L_{S U} \\
+ \begin{cases}10 \log _{10}\left(\left(\frac{d_{t}^{E_{T}}}{\left(x_{t} w_{r}\right)^{E_{S}}} \frac{4 \pi d_{r}}{\lambda}\right)^{E_{L}}\right), & \text { if } d_{r} \leq d_{b} \\
10 \log _{10}\left(\left(\frac{d_{t}^{E_{T}}}{\left(x_{t} w_{r}\right)^{E_{S}}} \frac{4 \pi d_{r}^{2}}{\lambda d_{b}}\right)^{E_{L}}\right), & \text { if } d_{r}>d_{b}\end{cases}
\end{gathered}
$$

where $d_{t} / d_{r}$ denotes the distance of the TX/RX to the intersection center, $w_{r}$ is width of the $\mathrm{RX}$ street, and $x_{t}$ is the distance of the TX to the wall, respectively.

As the NLOS path-loss at a certain distance can be calculated by the model, the NLOS received power $P_{R X}(d)$ for a certain $P_{T X}(d)$ can be calculated using the following expression,

$$
P_{R X}(d)=P_{T X}(d)-S_{L o s s}+G_{a}-P L\left(d_{r}, d_{t}, w_{r}, x_{t}, i_{s}\right)
$$

where $S_{\text {Loss }}$ and $G_{a}$ are the system loss and the combined TX/RX antenna gain, respectively. For the comparison both of these parameters are assumed to be zero as the effect of averaged antenna gain and cable losses have been removed from the measured channel gains before calculating the received powers. The NLOS model parameters and their values are listed in Table II.

TABLE II

NLOS PATH-LOSS MODEL PARAMETERS

\begin{tabular}{ll}
\hline Parameters & Values \\
\hline Curve shift, $C(\mathrm{~dB})$ & 3.75 \\
Sub-urban loss, $L_{S U}(\mathrm{~dB})$ & 2.94 \\
Urban loss factor, $i_{s}$ & 0 \\
Sub-urban loss factor, $i_{s}$ & 1 \\
Loss exponent, $E_{L}$ & 2.69 \\
Small-scale fading, $\sigma(\mathrm{dB})$ & 4.1 \\
Street exponent, $E_{S}$ & 0.81 \\
TX distance exponent, $E_{T}$ & 0.957 \\
Break even distance, $d_{b}(\mathrm{~m})$ & 180 \\
\hline
\end{tabular}

\section{VALIDATION OF THE NLOS MODEL}

In this section, the NLOS path-loss model is compared to the channel measurements data. It is desired to investigate whether the overall behavior of the received power in NLOS, both for the model and measurements, changes in accordance to the geometry of the respective intersection.

The NLOS $P_{R X}(d)$, obtained from the measurements and model, as a function of $d_{d c}$ for Intersections- 1 to 4 are presented in Fig. 3(a) to 3(d). The $P_{R X}(d)$ from the model is calculated by using the measured distances. Thus, the powers from each measurement and their respective powers from the model are plotted together to find out the difference.

From Figs. 3(a), 3(b) and 3(c), it can be seen that the measurement data fit very well to the NLOS model for Intersection-1, 2 and 3. However, in Intersection-2, the difference in the model and measurement starts to grow for 


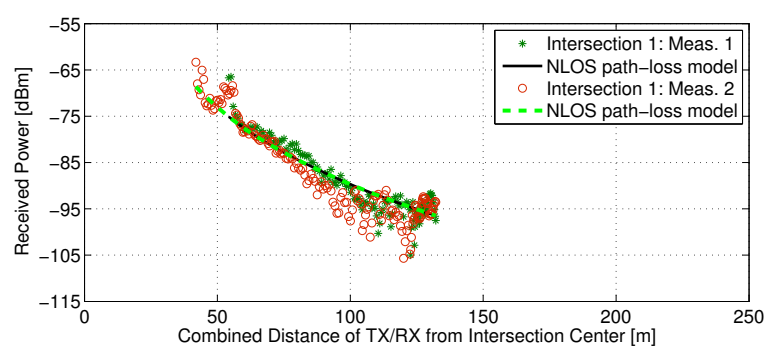

(a) Intersection-1

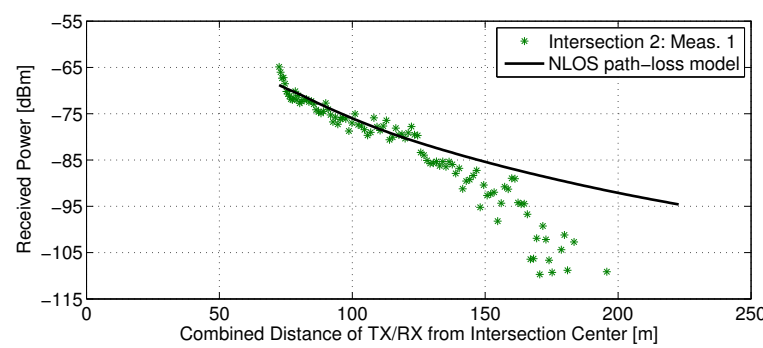

(b) Intersection-2

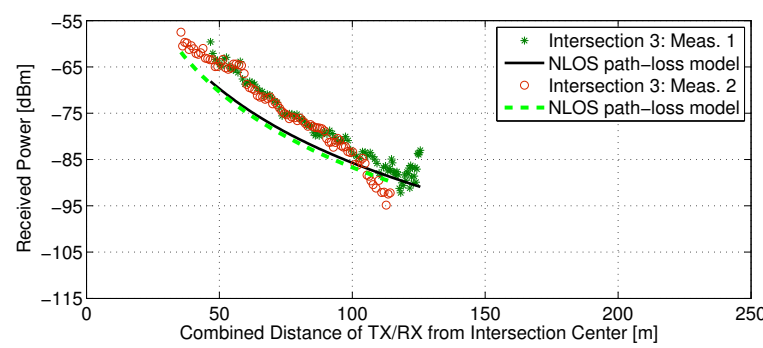

(c) Intersection-3

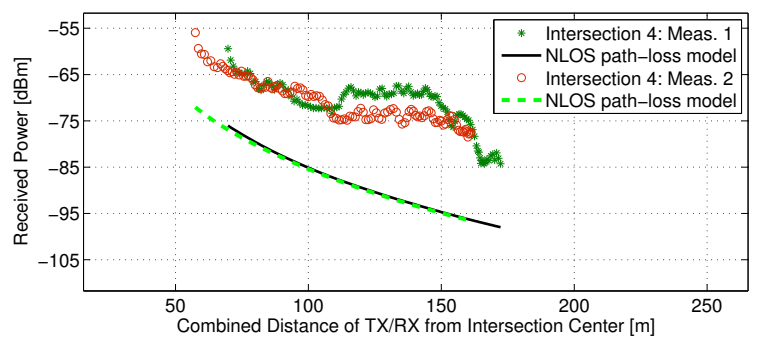

(d) Intersection-4

Fig. 3. Received power $P_{R X}(d)$ as a function of combined distance of TX/RX from the intersection center $\left(d_{d c}\right)$ for Intersection- 1 to Intersection- 4 : measurement and model.

$d_{d c}>130 \mathrm{~m}$. The reason behind this is the curved TX street. The TX street start to bend at an approximate distance of $60 \mathrm{~m}$ from the intersection center (see Fig. 1(b)). Due to that many significant MPCs, which are visible at $d_{d c}<130 \mathrm{~m}$, and start to disappear gradually for $d_{d c}>130$, which in turn continuously reduce the total $P_{R X}$. On the other hand, the model assumes the intersecting streets to be straight and perpendicular to each other, thus we do not see such power degradation in the model curve in Fig. 3(b) and that is one of the limitations in the model, urban streets are not always straight and perpendicular.

Another, interesting difference can be found in Fig. 3(d), where the difference in the measurement data and the model is quite large. After taking a closer look at the intersection and doing some visual inspection with the help of video recordings it is found that there are metallic window structures at the corner of the buildings that are situated at the corner of the street intersection right in front of the TX and RX. The strength of the reflected MPCs from these metallic window frames is very large, giving an unexpected rise to the received power. From the Fig. 3(d) we can see that the NLOS model is unable to capture this unusual behavior in the received power which is due to the material properties and the placement of scatterers. Although Mangel et. al. in [7] tried to select most representative street intersection in the city of Munich but still they may not be representative enough for many other cities. This causes another limitation in the model that the NLOS model cannot capture unusual behaviors due to the variety in the scattering environment of street intersections.

From this validation study it is found that it could be beneficial to add another intersection dependent gain parameter $\left(G_{I D}\right)$ in the model of (1) to compensate for the loss or gain in the power due to variations in the scattering as follows,

$$
\begin{gathered}
P L\left(d_{r}, d_{t}, w_{r}, x_{t}, i_{s}\right)=C+i_{s} L_{S U}+G_{I D} \\
+ \begin{cases}10 \log _{10}\left(\left(\frac{d_{t}^{E_{T}}}{\left(x_{t} w_{r}\right)^{E_{S}}} \frac{4 \pi d_{r}}{\lambda}\right)^{E_{L}}\right), & \text { if } d_{r} \leq d_{b} \\
10 \log _{10}\left(\left(\frac{d_{t}^{E_{T}}}{\left(x_{t} w_{r}\right)^{E_{S}}} \frac{4 \pi d_{r}^{2}}{\lambda d_{b}}\right)^{E_{L}}\right), & \text { if } d_{r}>d_{b}\end{cases}
\end{gathered}
$$

To find the correct values of such a parameter, available measurement data is not sufficient thus it is left for future investigations. After this investigation a need of new and larger amount of measurement data is felt to find a better and more generalized model for urban intersections.

In the comparison plots in Fig. 3(a) to 3(d) measured $P_{R X}$ data points deviated slightly from the model curves, which is due to the large-scale fading. Whereas the model curves are showing a distance dependent mean path-loss only, without effect of any fading. As mentioned earlier in the APDP derivation, the effect of small-scale fading has been removed by averaging the APDP but the effect of largescale fading is not removed and thus the measured $P_{R X}$ data points fluctuate around a distance dependent path-loss curves. Therefore, variations in the large scale fading for all intersections with respect to the model are investigated. The large-scale fading for each intersection is approximated as lognormal and the standard deviation $(\sigma)$ of zero mean Gaussian distributed large-scale fading is parameterized in Table III.

TABLE III

LARGE-SCALE FADING PARAMETER

\begin{tabular}{ll}
\hline Large-scale fading & $\sigma$ \\
\hline Intersection-1 $(\mathrm{dB})$ & 6.26 \\
Intersection-2 $(\mathrm{dB})$ & 5.5 \\
Intersection-3 $(\mathrm{dB})$ & 4.16 \\
Intersection-4 $(\mathrm{dB})$ & 3.2 \\
\hline
\end{tabular}




\section{SUMMARY AND CONCLUSIONS}

We present a validation of the NLOS path-loss model named VirtualSource 11p developed for vehicle-to-vehicle communications at $5.9 \mathrm{GHz}$. A large amount of measurement data collected at selected intersections in the city of Munich Germany is used to develop the model. In this paper the model is validated with the help of an independent measurement data set, recorded during the DRIVEWAY measurement campaign in the city of Lund and Malmö in southern Sweden. It is intended to test the model for its accuracy and flexibility. It is found that the model is very flexible and fits well to most of our measurements in different intersections. However, there are some cases in which the model does not fit well to the measurements due to rich scattering in some intersections. The results in this study suggest to introduce an intersection dependent gain parameter in the reference NLOS such that it can cope with the varying richness of scattering.

\section{REFERENCES}

[1] J. Karedal, F. Tufvesson, T. Abbas, O. Klemp, A. Paier, L. Bernadó, and A. F. Molisch, "Radio channel measurements at street intersections for vehicle-to-vehicle safety applications," in IEEE VTC 71st Vehicular Technology Conference (VTC 2010-spring), May 2010.

[2] T. Mangel, M. Michl, O. Klemp, and H. Hartenstein, "Real-world measurements of non-line-of-sight reception quality for $5.9 \mathrm{GHz}$ IEEE $802.11 \mathrm{p}$ at intersections," Communication Technologies for Vehicles, Springer Berlin Heidelberg, vol. 6596, pp. 189-202, 2011.

[3] M. Schack, J. Nuckelt, R. Geise, L. Thiele, and T. Kürner, "Comparison of path loss measurements and predictions at urban crossroads for C2C communications," in 5th European Conference on Antennas and Propagation (EuCAP), April 2011.

[4] T. Abbas, F. Tufvesson, and J. Karedal, "Measurement based shadow fading model for vehicle-to-vehicle network simulations," ArXiv e-prints, Mar. 2012

[5] J. Nuckelt, T. Abbas, F. Tufvesson, C. F. Mecklenbräuker, L. Bernado, and T. Kürner, "Comparison of ray tracing and channel-sounder measurements for vehicular communications," in 2013 IEEE 77th Vehicular Technology Conference: VTC2013-Spring, 2013-06-2. IEEE, 2013.

[6] T. Abbas, J. Kredal, and F. Tufvesson, "Measurement-Based Analysis The Effect of Complementary Antennas and Diversity on Vehicle-toVehicle Communication," IEEE Antennas and Wireless Propagation Letters, vol. 12, no. 1, pp. 309-312, 2013. [Online]. Available: http://lup.lub.lu.se/record/3516482/file/3555826.pdf

[7] T. Mangel, O. Klemp, and H. Hartenstein, " $5.9 \mathrm{GHz}$ inter-vehicle communication at intersections: a validated non-line-of-sight path-loss and fading model," EURASIP Journal on Wireless Communications and Networking, vol. 2011, no. 1, p. 182, 2011.

[8] A. Paier, L. Bernado, J. Karedal, O. Klemp, and A. Kwoczek, "Overview of vehicle-to-vehicle radio channel measurements for collision avoidance applications," in Vehicular Technology Conference (VTC 2010-Spring), 2010 IEEE 71st, 2010, pp. 1-5.

[9] Google earth v7.1.1.1888 (2013). [Online]. Available: http://www.google.com/earth/index.html [Accessed: 2013/08/15]

[10] A. Thiel, O. Klemp, A. Paier, L. Bernadó, J. Karedal, and A. Kwoczek, "In-situ vehicular antenna integration and design aspects for vehicle-tovehicle communications," in EUCAP, Apr. 2010.

[11] R. Thoma, D. Hampicke, A. Richter, G. Sommerkorn, A. Schneider, U. Trautwein, and W. Wirnitzer, "Identification of time-variant directional mobile radio channels," Instrumentation and Measurement, IEEE Transactions on, vol. 49, no. 2, pp. 357 -364, apr 2000.

[12] IEEE, "Part 11: Wireless lan medium access control (MAC) and physical layer (PHY) specifications: Amendment 7: Wireless access in vehicular environment," IEEE 802.11p,2010, 2010.

[13] H. El-Sallabi, "Fast path loss prediction by using virtual source technique for urban microcells," in IEEE 51st Vehicular Technology Conference Proceedings (VTC 2000-Spring) Tokyo., vol. 3, 2000, pp. 2183 -2187 vol.3. 